HU-EP-05/29

\title{
Oscillator quantization of the massive scalar particle dynamics on AdS spacetime
}

\author{
Harald Dorn ${ }^{a}$ and George Jorjadze ${ }^{b}$ \\ ${ }^{a}$ Institut für Physik der Humboldt-Universität zu Berlin, \\ Newtonstraße 15, D-12489 Berlin, Germany \\ ${ }^{b}$ Razmadze Mathematical Institute, \\ M.Aleksidze 1, 0193, Tbilisi, Georgia
}

\begin{abstract}
The set of trajectories for massive spinless particles on $A d S_{N+1}$ spacetime is described by the dynamical integrals related to the isometry group $S O(2, N)$. The space of dynamical integrals is mapped one to one to the phase space of the $N$-dimensional oscillator. Quantizing the system canonically, the classical expressions for the symmetry generators are deformed in a consistent way to preserve the $s o(2, N)$ commutation relations. This quantization thus yields new explicit realizations of the spin zero positive energy UIR's of $S O(2, N)$ for generic $N$. The representations as usual can be characterized by their minimal energy $\alpha$ and are valid in the whole range of $\alpha$ allowed by unitarity.
\end{abstract}

Keywords: AdS space; $S O(2, N)$ group; geometric and canonical quantization;

PACS: 11.10. EF; 11.10. Kk; 11.10. Lm; 11.25. Hf 


\section{Introduction}

$A d S$ spacetimes have an isometry group of the same dimension as the corresponding Minkowski case. Therefore, quantization of particle dynamics within standard Hamiltonian reduction techniques is possible. This has been done before for lower dimensional cases [1, 2, 3]. Our present paper continues a systematic study of both the classical and quantum particle dynamics on $A d S_{N+1}$ for generic $N$ started in [4]. With these investigations we find new explicit realizations of UIR's of $S O(2, N)$.

Another motivation for this program comes from possible interrelations with the corresponding quantum field theories and string theories on $A d S_{N+1}$, which play a crucial role in the $A d S / C F T$ correspondence, see e.g. [5, 6, 7]. In this context one of the most challenging open problems is the quantization of strings on $A d S_{5} \times S^{5}$. Certainly, the full understanding of quantized particle dynamics on such backgrounds could be a useful warm-up.

There are well-known explicit expressions for field theoretical propagators on $A d S$ spacetime. They are crucial tools for the use of the $A d S / C F T$ correspondence in application to the large $N$ and strong 't Hooft coupling limit on the gauge field theory side. Less is known about explicit expressions for field theoretical propagators on $A d S \times S$ backgrounds [7]. Since they could be very helpful to understand the fate of the holographic picture in the BMN limit [8], any technique for constructing propagators should be of interest. In [4] we have explicitely shown that at least some of the standard $A d S$ propagators can be obtained as propagation kernels of the quantized particle.

Of particular interest is also the case of massless particles [9], which in field theory have been related to singleton representations of the conformal group. For them it is natural to relate the original spacetime to the conformal boundary of a spacetime with just one more dimension.

In [4], quantizing the dynamics of a massive scalar particle in $A d S_{N+1}$ space-time along the lines of geometric quantization, we have constructed the following representations of the isometry group generating $s o(2, N)$ algebra

$$
\begin{array}{lc}
E=\alpha+\zeta_{l} \partial_{\zeta_{l}}, & J_{m n}=i\left(\zeta_{n} \partial_{\zeta_{m}}-\zeta_{m} \partial_{\zeta_{n}}\right) \\
z_{n}^{*}=2 \alpha \zeta_{n}+\left(2 \zeta_{n} \zeta_{l}-\zeta^{2} \delta_{n l}\right) \partial_{\zeta_{l}}, & z_{n}=\partial_{\zeta_{n}}, \quad(l, m, n=1, \ldots, N)
\end{array}
$$

Here $E$ and $J_{m n}$ are the generators of the compact subgroup $S O(2) \times S O(N)$, while $z_{n}^{*}$ and $z_{n}$ stand for the two kind of boosts of the theory. The representations (1.1) are characterized by 
the parameter $\alpha$, which corresponds to the lowest eigenvalue of the energy operator $E$. The Hilbert space is spanned by the holomorphic functions of $N$ complex variables $\zeta_{n}(n=1, \ldots, N)$ defined inside some bounded domain and the measure for the scalar product depends on the parameter $\alpha$ and the dimension $N$. The case $N=1$ corresponds to Bargmann's representation of the $s o(2,1)$ algebra given on holomorphic functions inside the unit disk $|\zeta|<1$ (see e.g. [10]), and the regularity of the scalar product requires $\alpha>1 / 2$. For $N>1$ the integration domain is more complicated and the integration measure turns out to be regular for $\alpha>N-1$ only.

Since the constructed Hilbert space seems to have some similarities to the Fock space of a $N$-dimensional oscillator, it is natural to ask for a corresponding explicit construction in terms of oscillator variables and its canonical quantization. From [4] there remained also another puzzling question. The allowed values of $\alpha$ do not exhaust the well-known unitarity bound, see e.g. [1] and refs. therein,

$$
\alpha \geq(N-2) / 2 \text { for } N \geq 2 \text { and } \quad \alpha \geq 0 \text { for } N=1 \text {. }
$$

In addition, within the representations (1.1) one cannot reach values for $\alpha$, corresponding to the Casimir number $C=\left(1-N^{2}\right) / 4$ related to a conformal invariant setting in $A d S_{N+1}$.

The formulation in terms of oscillator variables for the simplest case $N=1$ can be obtained by the Holstein-Primakoff method [12] in the form

$$
z=\sqrt{H+2 \alpha} a, \quad z^{*}=a^{*} \sqrt{H+2 \alpha}, \quad E=H+\alpha,
$$

where $H$ is the normal ordered oscillator Hamiltonian $H=a^{*} a$ and the square root of the positive operator $H+2 \alpha$ is defined in standard manner via spectral representation. The representation (1.3) is unitary and irreducible for $\alpha>0$. Thus, the canonical scheme covers all lowest weight UIR's of the $s o(2,1)$ algebra. The aim of the present paper is to generalize this result to arbitrary $N$.

\section{Classical theory}

The $N+1$ dimensional $A d S$ space can be represented as the universal covering of the hyperboloid of radius $R$

$$
X_{0}^{2}+X_{0^{\prime}}^{2}-\sum_{n=1}^{N} X_{n}^{2}=R^{2}
$$


embedded in the $N+2$ dimensional flat space $\mathbb{R}_{N}^{2}$ with coordinates $X_{A}, A=\left(0,0^{\prime}, 1, \ldots, N\right)$ and the metric tensor $G_{A B}=\operatorname{diag}(+,+,-, \ldots,-)$. The induced metric tensor on the hyperboloid has Lorentzian signature and the polar angle $\theta$ in the $\left(X_{0}, X_{0^{\prime}}\right)$ plane can be taken as the time coordinate: $X_{0}=r \cos \theta, X_{0^{\prime}}=r \sin \theta \quad\left(r^{2}=X_{n} X_{n}+R^{2}\right)$.

The dynamics of a massive particle moving on the hyperboloid we describe by the action

$$
S=-\int d \tau\left[m \sqrt{\dot{X}^{A} \dot{X}_{A}}+\frac{\mu}{2}\left(X^{A} X_{A}-R^{2}\right)\right],
$$

where $m>0$ is the particle mass, $\mu$ is a Lagrange multiplier and $\tau$ is an evolution parameter. The time direction we fix in a $S O(2, N)$-invariant way by requiring $\dot{\theta}>0$, which is equivalent to $X_{0} \dot{X}_{0^{\prime}}-X_{0^{\prime}} \dot{X}_{0}>0$. The space-time isometry group $S O(2, N)$ provides the Noether conserved quantities

$$
J_{A B}=P_{A} X_{B}-P_{B} X_{A},
$$

where $P_{A}$ are the canonical momenta. Since $\theta$ is the time coordinate, $J_{00^{\prime}}$ is associated with the particle energy. We denote it by $E$ and due to the choice of the time direction it is positive

$$
E=P_{0} X_{0^{\prime}}-P_{0^{\prime}} X_{0}=\frac{m}{\sqrt{\dot{X}^{A} \dot{X}_{A}}}\left(X_{0} \dot{X}_{0^{\prime}}-X_{0^{\prime}} \dot{X}_{0}\right)>0 .
$$

The conservation of $J_{A B}$ allows to represent the set of trajectories geometrically without solving the dynamical equations. From Eq. (2.3) we find $\mathrm{N}$ equations as identities in the variables $(P, X)$

$$
E X_{n}=J_{0 n} X_{0^{\prime}}-J_{0^{\prime} n} X_{0}, \quad(n=1, \ldots, N) \text {. }
$$

Since $E, J_{0^{\prime} n}, J_{0^{\prime} n}$ are constants, Eq. (2.5) defines a 2-dimensional plane in the embedding space $\mathbb{R}_{N}^{2}$ and the intersection of this plane with the hyperbola (2.1) is a particle trajectory.

The action (2.2) is invariant under the reparametrizations $\tau \rightarrow f(\tau)$ with the corresponding transformations of the Lagrange multiplier $\left(\mu \rightarrow \mu / f^{\prime}\right)$. The gauge symmetry, as usual, leads to the dynamical constraints. Applying Dirac's procedure, we find three constraints

$$
X^{A} X_{A}-R^{2}=0, \quad P_{A} P^{A}-m^{2}=0, \quad P_{A} X^{A}=0
$$

which fix the quadratic Casimir number of the symmetry group

$$
C=\frac{1}{2} J_{A B} J^{A B}=m^{2} R^{2}
$$

For further calculations it is convenient to introduce complex valued dynamical integrals

$$
z_{n}=J_{0^{\prime} n}-i J_{0 n}, \quad z_{n}^{*}=J_{0^{\prime} n}+i J_{0 n}
$$


and the scalar variables $\lambda^{2}=z_{n}^{*} z_{n}, \rho^{4}=z^{* 2} z^{2}\left(\right.$ with $z^{2}=z_{n} z_{n}$ ). Then (2.7) becomes

$$
E^{2}+J^{2}=\lambda^{2}+\alpha^{2}
$$

where

$$
J^{2}=\frac{1}{2} J_{m n} J_{m n} \quad \text { and } \quad \alpha=m R .
$$

A set of other quadratic relations follows from (2.3) as identities in the variables $(P, X)$

$$
J_{A B} J_{A^{\prime} B^{\prime}}=J_{A A^{\prime}} J_{B B^{\prime}}-J_{A B^{\prime}} J_{B A^{\prime}} .
$$

These equations are nontrivial in terms of the dynamical integrals, if all indices $A, B, A^{\prime}, B^{\prime}$ are different. Taking $A=0, B=0^{\prime}, A^{\prime}=m$ and $B^{\prime}=n(m \neq n)$ we obtain

$$
2 i E J_{m n}=z_{m}^{*} z_{n}-z_{n}^{*} z_{m}
$$

and its square yields $4 E^{2} J^{2}=\lambda^{4}-\rho^{4}$. Then, together with (2.9) we conclude that $E^{2}$ and $J^{2}$ are roots of the quadratic equation $4 x^{2}-4\left(\lambda^{2}+\alpha^{2}\right) x+\lambda^{4}-\rho^{4}=0$ and find

$$
E^{2}=\frac{1}{2}\left(\lambda^{2}+\alpha^{2}+\sqrt{\alpha^{4}+2 \alpha^{2} \lambda^{2}+\rho^{4}}\right) .
$$

We neglect the second root as unphysical, since it does not provide positivity of the energy (see [4] for more details). According to (2.13) $\alpha$ is the lowest value of energy. Eqs. (2.13) and (2.12) define $E$ and $J_{m n}$ as functions of $\left(z_{n}, z_{n}^{*}\right)$ and, therefore, $\left(z_{n}, z_{n}^{*}\right)$ are global coordinates on the space of dynamical integrals, which is the physical phase space $\Gamma_{p h}$ of the system.

Based on the canonical brackets between the variables $(P, X)$ the $s o(2, N)$ Poisson bracket algebra of the symmetry generators can be written as

$$
\begin{aligned}
& \left\{z_{m}^{*}, z_{n}\right\}=2 J_{m n}-2 i \delta_{m n} E, \quad\left\{z_{m}, z_{n}\right\}=0=\left\{z_{m}^{*}, z_{n}^{*}\right\} \\
& \left\{J_{l m}, z_{n}\right\}=z_{l} \delta_{m n}-z_{m} \delta_{l n}, \quad\left\{E, z_{n}\right\}=-i z_{n}, \quad\left\{E, J_{m n}\right\}=0
\end{aligned}
$$

and $J_{m n}$ 's form the $s o(N)$ algebra. These relations are preserved after reduction to $\Gamma_{p h}$, since the constraints (2.6) are $S O(2, N)$ scalars. But (2.14)-(2.15) become essentially non-linear in terms of the independent variables $z_{n}, z_{n}^{*}$ and their quantum realization is a nontrivial issue.

Our aim is to find a canonical parametrization of $\Gamma_{p h}$ and then to quantize the system canonically. For this purpose we introduce a set of $2 N$ variables $\left(a_{n}, a_{n}^{*} ; n=1, \ldots, N\right)$ with the canonical Poisson brackets

$$
\left\{a_{n}, a_{m}\right\}=0=\left\{a_{n}^{*}, a_{m}^{*}\right\}, \quad\left\{a_{n}, a_{m}^{*}\right\}=i \delta_{m n}
$$


and assume that the generators of the compact transformations are given by

$$
E=H+\alpha, \quad J_{m n}=i\left(a_{n}^{*} a_{m}-a_{m}^{*} a_{n}\right)
$$

where $H=a_{n}^{*} a_{n}$ is the oscillator Hamiltonian. Note that Eqs. (2.16)-(2.17) immediately provide the $s o(2) \times s o(N)$ part of $s o(2, N)$ algebra and the $s o(N)$ scalar (2.10) constructed from $J_{m n}$ becomes $J^{2}=H^{2}-a^{* 2} a^{2}$, with $a^{2}=a_{n} a_{n}$ and $a^{* 2}=a_{n}^{*} a_{n}^{*}$.

We look now for a parametrization of the generators $z_{n}$ and $z_{n}^{*}$ in the form

$$
z_{n}=X\left(H, J^{2}\right) a_{n}+Y\left(H, J^{2}\right) a^{2} a_{n}^{*}, \quad z_{n}^{*}=X\left(H, J^{2}\right) a_{n}^{*}+Y\left(H, J^{2}\right) a^{* 2} a_{n}
$$

where $X$ and $Y$ are real functions of the scalar variables $\left(H, J^{2}\right)$. This ansatz guarantees the correct commutation relations between compact and non-compact generators. The Casimir condition (2.9) and the quadratic relation (2.12) yield two equations for the functions $X, Y$

$$
\begin{aligned}
& H X^{2}+\left(H^{2}-J^{2}\right)\left(2 X Y+H Y^{2}\right)=H^{2}+J^{2}+2 \alpha H \\
& X^{2}-\left(H^{2}-J^{2}\right) Y^{2}=2(H+\alpha)
\end{aligned}
$$

After elimination of $X$, (2.19) reduces to a quadratic equation for $Y^{2}$. Choosing the root which is nonsingular for $J^{2}=0$ and putting $Y=-\sqrt{Y^{2}}$, we obtain

$$
X=-\frac{1}{2 Y}\left[1+2 H Y^{2}\right], \quad Y=-\left(2 H+4 \alpha+2 \sqrt{(H+2 \alpha)^{2}-J^{2}}\right)^{-\frac{1}{2}} .
$$

Note that the choice $Y=\sqrt{Y^{2}}$ leads to a canonically equivalent answer, reproduced from (2.18) by the inversion $a_{n} \mapsto-a_{n}$.

Due to (2.18) and (2.20) the $S O(N)$ scalars $z^{2}$ and $a^{2}$ are related by

$$
z^{2}=F a^{2}
$$

where $F$ is the following function of real scalar variables

$$
F=\sqrt{(H+2 \alpha)^{2}-J^{2}}=\sqrt{(E+\alpha)^{2}-J^{2}} .
$$

This function also played an important role in the scheme of geometric quantization [4]. Note that the parametrization of $\Gamma_{p h}$ given by (2.18) and (2.20) can be written as

$$
z_{n}=\frac{1}{\sqrt{2(H+2 \alpha+F)}}\left((2 H+2 \alpha+F) a_{n}-a^{2} a_{n}^{*}\right) \text {. }
$$


The relation (2.21) helps to invert (2.18) and we find

$$
a_{n}=\frac{1}{2 E}\left(X z_{n}-\frac{Y}{F} z^{2} z_{n}^{*}\right), \quad a_{n}^{*}=\frac{1}{2 E}\left(X z_{n}^{*}-\frac{Y}{F} z^{* 2} z_{n}\right),
$$

where now $X, Y$ and $F$ are treated as functions of $\left(E, J^{2}\right)$ and they are obtained from (2.20) replacing $H$ by $E-\alpha$.

The direct calculation shows that the canonical brackets (2.16) lead to the $s o(2, N)$ algebra (2.14)-(2.15) . To our knowledge, the constructed canonical parametrization and its quantum realization for generic $N$ was not discussed in the literature before.

\section{Quantum theory}

We quantize the $A d S$ particle dynamics using the canonical coordinates constructed in the previous section. We will use for quantum operators the same letters as for the corresponding classical variables. Canonical quantization assumes a realization of the canonical commutation relations

$$
\left[a_{n}, a_{m}\right]=0=\left[a_{n}^{*}, a_{m}^{*}\right], \quad\left[a_{n}, a_{m}^{*}\right]=\delta_{m n},
$$

and a construction of the symmetry generators $E, J_{m n}, z_{n}, z_{n}^{*}$ on the basis of the parametrization (2.17), (2.18) and (2.20). Due to the non-linearity of the parametrization we face with the problem of ordering ambiguity.

There is not such a problem for the generators of $S O(N)$ rotations

$$
J_{m n}=i\left(a_{n}^{*} a_{m}-a_{m}^{*} a_{n}\right)
$$

and the operator $J^{2}$ in terms of the creation-annihilation operators becomes

$$
J^{2}=\frac{1}{2} J_{m n} J_{m n}=H^{2}+(N-2) H-a^{* 2} a^{2}
$$

where $H=a_{n}^{*} a_{n}$ is the normal ordered oscillator Hamiltonian, $a^{2}=a_{n} a_{n}$ and $a^{* 2}=a_{n}^{*} a_{n}^{*}$.

Classically we had for the quadratic Casimir $C=m^{2} R^{2}=\alpha^{2}$. In the quantum case we continue to denote the lowest energy value by $\alpha$, i.e.

$$
E=H+\alpha
$$

but we have to expect a renormalization of the relation of $\alpha$ to the quadratic Casimir, which defines the squared mass of the quantum particle. Since we are interested in representations 
which are unitary equivalent to those constructed by geometric quantization we take the corresponding renormalized relation from [4]

$$
C=E^{2}+\frac{1}{2} J_{m n} J_{m n}-\frac{1}{2}\left(z_{n}^{*} z_{n}+z_{n} z_{n}^{*}\right)=\alpha(\alpha-N) \text {. }
$$

Note in addition, that the quadratic relation (2.12) due to (1.1) is deformed into

$$
z_{m}^{*} z_{n}-z_{n}^{*} z_{m}=2 i(E-1) J_{m n}, \quad \text { or } \quad z_{n} z_{m}^{*}-z_{m} z_{n}^{*}=2 i(E+1) J_{m n}
$$

The ordering problem is more complicated for $z_{n}$ and $z_{n}^{*}$. We look for them in the form

$$
z_{n}=X_{N}\left(H, J^{2}\right) a_{n}+Y_{N}\left(H, J^{2}\right) a^{2} a_{n}^{*}, \quad z_{n}^{*}=a_{n}^{*} X_{N}\left(H, J^{2}\right)+a_{n} a^{* 2} Y_{N}\left(H, J^{2}\right),
$$

where $X_{N}$ and $Y_{N}$ are real unknown functions of the commuting operators $H$ and $J^{2}$, similarly to (2.18). Due to the ordering ambiguity, the functions $X_{N}, Y_{N}$ are quantum mechanical deformations of $X, Y$ and the index $N$ indicates that the deformations can depend on the dimension $N$. To fix these functions we use the quantum versions of the Casimir condition (3.5) and the quadratic relation (3.6), which can be written as

$$
z_{n} z_{n}^{*}=H^{2}+J^{2}+(2 \alpha+N) H+2 N \alpha \quad \text { and } \quad z_{n} z_{m}^{*}-z_{m} z_{n}^{*}=2(H+\alpha+1)\left(a_{m}^{*} a_{n}-a_{n}^{*} a_{m}\right)
$$

respectively. Taking into account the commutation relations between the scalar operators

$$
\left[H, a^{2}\right]=-2 a^{2}, \quad\left[H, a^{* 2}\right]=2 a^{* 2}, \quad\left[a^{2}, a^{* 2}\right]=4 H+2 N,
$$

from (3.8) we find two equations for $X_{N}, Y_{N}$

$$
\begin{gathered}
(H+N) X_{N}^{2}+\left(H^{2}-J^{2}+(N+2) H+2 N\right)\left(2 X_{N} Y_{N}+(H+2) Y_{N}^{2}\right)= \\
H^{2}+J^{2}+(2 \alpha+N) H+2 \alpha N, \\
X_{N}^{2}-\left(H^{2}-J^{2}+(N+2) H+2 N\right) Y_{N}^{2}=2(H+\alpha+1) .
\end{gathered}
$$

These equations contain only commuting operators and they can be solved as algebraic equations like (2.19) in the classical case. After elimination of $X_{N}$, (3.10) reduces to a quadratic equation for $Y_{N}^{2}$. Neglecting the root, which corresponds to the singular solution in the classical limit, and choosing $Y_{N}=-\sqrt{Y_{N}^{2}}$, we obtain (see (2.20) $)$

$$
\begin{aligned}
& X_{N}=-\frac{1}{2 Y_{N}}\left[1+(2 H+N+2) Y_{N}^{2}\right] \\
& Y_{N}=-\left(2 H+4 \alpha-N+2+2 \sqrt{(H+2 \alpha)^{2}-(N-2)(H+2 \alpha)-J^{2}}\right)^{-\frac{1}{2}} .
\end{aligned}
$$


These operator expressions are naturally defined on the eigenstates of $H$ and $J^{2}$ as multiplication operators. The choice $Y_{N}=\sqrt{Y_{N}^{2}}$ leads to an unitary equivalent answer, since the change of sign of $Y_{N}$ corresponds to the inversion $a_{n} \rightarrow-a_{n}$.

Eqs. (3.7) and (3.11) provide a deformed version of (2.23)

$$
\begin{array}{lc}
z_{n}=\frac{1}{\sqrt{2\left(H_{N}+2 \alpha_{N}+F_{N}\right)}}\left(\left(2 H_{N}+2 \alpha_{N}+F_{N}\right) a_{n}-a^{2} a_{n}^{*}\right), \quad \text { with } \\
F_{N}=\sqrt{\left(H_{N}+2 \alpha_{N}\right)^{2}-J_{N}^{2}}, & \alpha_{N}=\alpha-\frac{N}{2}, \\
H_{N}=H+\frac{N+2}{2}, & J_{N}^{2}=J^{2}+\left(\frac{N-2}{2}\right)^{2} .
\end{array}
$$

The notation in the above formulas has been chosen in a form to make both the structural similarities as well as the modifications relative to their classical counterparts (2.22)-(2.23) manifest. One can check that for $N=1 \mathrm{Eq}$. (3.12) reproduce the operator $z$ in (1.3).

The ansatz (3.7) obviously satisfies the commutation relations of $z_{n}$ with $E$ and $J_{m n}$ for arbitrary $X_{N}$ and $Y_{N}$

$$
\left[E, z_{n}\right]=-z_{n}, \quad\left[J_{l m}, z_{n}\right]=i\left(\delta_{l n} z_{m}-\delta_{m n} z_{l}\right)
$$

To calculate the commutation relations between the operators (3.7) we use the exchange relations of the creation-annihilation operators with the scalar operators. These relations are derived in the Appendix and by (A.10)-(A.17) we find an alternative form of (3.7)

$$
\begin{gathered}
z_{n}=a_{n} \tilde{X}_{N}+a_{n}^{*} a^{2} \tilde{Y}_{N}, \quad z_{n}^{*}=\tilde{X}_{N} a_{n}^{*}+\tilde{Y}_{N} a^{* 2} a_{n}, \quad \text { where } \\
\tilde{X}_{N}=-\frac{1}{2 \tilde{Y}_{N}}\left(1+(2 H+N-2) \tilde{Y}_{N}^{2}\right), \\
\tilde{Y}_{N}=-\left(2 H+4 \alpha-N-2+2 \sqrt{(H+2 \alpha)^{2}-(N+2)(H+2 \alpha)+2 N-J^{2}}\right)^{-\frac{1}{2}} .
\end{gathered}
$$

Note that the functions (3.11) and (3.16) are related by

$$
\tilde{X}_{N}\left(H, J^{2}\right)=X_{N}\left(H-2, J^{2}\right), \quad \tilde{Y}_{N}\left(H, J^{2}\right)=Y_{N}\left(H-2, J^{2}\right) .
$$

The operators (3.15)-(3.16) satisfy the equations

$$
z_{n}^{*} z_{n}=H^{2}+J^{2}+(2 \alpha-N) H \quad \text { and } \quad z_{m}^{*} z_{n}-z_{n}^{*} z_{m}=2(H+\alpha-1)\left(a_{m}^{*} a_{n}-a_{n}^{*} a_{m}\right)
$$

which are equivalent to (3.5) and (3.6), respectively. 
It remains to check the commutation relations

$$
\left[z_{m}, z_{n}\right]=0, \quad\left[z_{m}^{*}, z_{n}\right]=2\left(a_{n}^{*} a_{m}-a_{m}^{*} a_{n}\right)-2 \delta_{m n}(H+\alpha)
$$

Using the two representations of $z_{n}$ operators (3.7) and (3.15), we obtain (see Appendix)

$$
\begin{aligned}
& {\left[z_{m}, z_{n}\right]=\left(a_{m} a_{n}^{*}-a_{n} a_{m}^{*}\right) a^{2} U\left(H, J^{2}\right), \quad \text { where }} \\
& U=X_{N}\left(H-2, J^{2}\right) \tilde{Y}_{N}\left(H, J^{2}\right)-Y_{N}\left(H-2, J^{2}\right) \tilde{X}_{N}\left(H, J^{2}\right),
\end{aligned}
$$

and due to (3.17), the commutator $\left[z_{m}, z_{n}\right]$ vanishes.

Calculating $\left[z_{m}^{*}, z_{n}\right]$ in a similar way we get the following structure (see Appendix)

$$
\left[z_{m}^{*}, z_{n}\right]=\delta_{m n} U_{0}+a_{m}^{*} a_{n} U_{1}+a_{n}^{*} a_{m} U_{2}+a_{m}^{*} a_{n}^{*} a^{2} U_{3}+a_{m} a_{n} a^{* 2} U_{4}
$$

where $U_{0}, \ldots, U_{4}$ are functions of the scalar variables $H, J^{2}$ like $U$ in (3.20). The exchange relations (A.10) and (A.19) provide $U_{3}=U_{4}=0, U_{2}=-U_{1}=2, U_{0}=-2(H+\alpha)$ and this completes the proof of (3.19).

The unitarity of our representations is guaranteed as long as $z_{n}$ and $z_{n}^{*}$ expressed by (3.7) in terms of $a_{n}$ and $a_{n}^{*}$ are adjoint to each other. This in turn implies selfadjoint $X_{N}$ and $Y_{N}$, hence positivity of the operator expression under the square root in (3.11). With the help of (3.3) this condition can be written as $a^{* 2} a^{2}+2(H+\alpha)(2 \alpha-N+2) \geq 0$ and it reproduces the unitarity bound (1.2).

Irreducibility of the representations is certainly given as long as the creation-annihilation operators $a_{n}^{*}$ and $a_{n}$ can be expressed in terms of the symmetry generators. This requires an inversion of (3.7). As a first step for this inversion we need the quantum analog of (2.21), which is obtained from (3.7) and (3.15)

$$
z^{2}=F_{N}\left(H, J^{2}\right) a^{2}
$$

Based on this relation we get finally

$$
a_{n}=\frac{1}{2(E+1)}\left(X_{N} z_{n}-\frac{Y_{N}}{F_{N}} z^{2} z_{n}^{*}\right), \quad a_{n}^{*}=\frac{1}{2 E}\left(z_{n}^{*} X_{N}-z_{n} z^{* 2} \frac{Y_{N}}{F_{N}}\right) .
$$

This inversion formula is well-defined as long as $\alpha$ is above the unitarity bound. But note also that for $\alpha$ just on the unitarity bound $F_{N}=\sqrt{a^{* 2} a^{2}}$, which cannot be inverted within the full oscillator space. Therefore irreducibility is lost at the unitarity bound. 


\section{Conclusions}

Our main result is the realization of spin zero $s o(2, N)$ representations in terms of just one set of $N$-dimensional oscillator operators. The formulas defining this representation are (3.2), (3.4), (3.7) and (3.11) (or equivalently (3.12) and (3.13)) and they reproduces the well-known unitarity bound (1.2).

The whole construction was based on an one to one map of the space of dynamical integrals of a scalar massive particle in $A d S_{N+1}$ to the phase space of a $N$-dimensional oscillator. This distinguishes our approach from the oscillator like representations of non-compact groups, developed in [13], where, using more oscillators, all symmetry generators are represented bilinearly in the oscillators operators and the representation space is selected out from the oscillator Fock space by some conditions, which can be interpreted as coherent state constraints.

Our $s o(2, N)$ representations arose as a by-product of scalar particle dynamics. It would be interesting to extend these considerations to particles with spin and to construct the corresponding $s o(2, N)$ representations with non-zero $s o(N)$ weights.

In a forthcoming paper [9] we will analyze in detail the quantization of massless particles. This will correspond to the special case $\alpha=(N \pm 1) / 2$ in which the symmetry group is enlarged to the conformal group of $A d S_{N+1}$. There we further comment on the reducibility of our representation of $s o(2, N)$ at the unitarity bound and discuss its relation to the singleton representations [14] and to massless particle dynamics in one dimension less, i.e. in $A d S_{N}$.

Acknowledgments. We thank H. Nicolai for discussions. G.J. is grateful to Humboldt University and AEI Potsdam for hospitality. His research was supported by grants from the DFG, GRDF and GAS. H.D. was supported in part by DFG with the grant DO 447-3/3.

\section{Appendix}

Let us consider the operators $H_{N}$ and $J_{N}^{2}$ given by (3.13), (3.3) and calculate their exchange relations with the operators $a_{n}$ and $b_{n}=a^{2} a_{n}^{*}$. The canonical commutators (3.1) provide

$$
\begin{array}{ll}
H_{N} a_{n}=a_{n}\left(H_{N}-1\right), & J_{N}^{2} a_{n}=a_{n}\left(J_{N}^{2}-2 H_{N}+1\right)+2 b_{n}, \\
H_{N} b_{n}=b_{n}\left(H_{N}-1\right), & J_{N}^{2} b_{n}=b_{n}\left(J_{N}^{2}+2 H_{N}+1\right)-2 a_{n}\left(H_{N}^{2}-J_{N}^{2}\right) .
\end{array}
$$

These relations are diagonalized by the operators

$$
c_{n}=b_{n}-a_{n}\left(H_{N}+J_{N}\right), \quad d_{n}=b_{n}-a_{n}\left(H_{N}-J_{N}\right)
$$


in the following form

$$
\begin{array}{ll}
H_{N} c_{n}=c_{n}\left(H_{N}-1\right), & J_{N}^{2} c_{n}=c_{n}\left(J_{N}-1\right)^{2} \\
H_{N} d_{n}=d_{n}\left(H_{N}-1\right), & J_{N}^{2} d_{n}=d_{n}\left(J_{N}+1\right)^{2} .
\end{array}
$$

Here $J_{N}=\sqrt{J_{N}^{2}}$ and the square root from the positive operator is defined in a standard way. Introducing new scalar variables

$$
u=H_{N}+J_{N} \quad \text { and } \quad v=H_{N}-J_{N},
$$

the functions (3.11) can be written as

$$
X_{N}=\frac{u \sqrt{u+\beta}-v \sqrt{v+\beta}}{u-v}, \quad Y_{N}=-\frac{\sqrt{u+\beta}-\sqrt{v+\beta}}{u-v}, \quad \text { with } \beta=2 \alpha-N .
$$

Due to (A.4)- (A.5), a function $f(u, v)$ satisfies the exchange relations

$$
f(u, v) c_{n}=c_{n} f(u-2, v), \quad f(u, v) d_{n}=d_{n} f(u, v-2) .
$$

Inverting (A.3)

$$
a_{n}=d_{n} \frac{1}{u-v}-c_{n} \frac{1}{u-v}, \quad b_{n}=d_{n} \frac{u}{u-v}-c_{n} \frac{v}{u-v}
$$

and using (A.8), we obtain

$$
\begin{aligned}
& f(u, v) a_{n}=a_{n} \frac{u f(u-2, v)-v f(u, v-2)}{u-v}-b_{n} \frac{f(u-2, v)-f(u, v-2)}{u-v}, \\
& f(u, v) b_{n}=a_{n} \frac{u v f(u-2, v)-u v f(u, v-2)}{u-v}-b_{n} \frac{v f(u-2, v)-u f(u, v-2)}{u-v} .
\end{aligned}
$$

Applying these exchange relations to the functions (A.7) we get

$$
\begin{aligned}
& X_{N} a_{n}=a_{n} X_{(1)}+b_{n} Y_{(1)}, \quad Y_{N} b_{n}=a_{n} X_{(2)}+b_{n} Y_{(2)}, \\
& X_{N} b_{n}=a_{n} X_{(3)}+b_{n} Y_{(3)}, \quad Y_{N} a_{n}=a_{n} X_{(4)}+b_{n} Y_{(4)},
\end{aligned}
$$

where

$$
\begin{aligned}
& X_{(1)}=\frac{u(u-2) \sqrt{u-2+\beta}-u v \sqrt{v+\beta}}{(u-v)(u-2-v)}-\frac{u v \sqrt{u+\beta}-v(v-2) \sqrt{v-2+\beta}}{(u-v)(u-v+2)}, \\
& Y_{(1)}=-\frac{(u-2) \sqrt{u-2+\beta}-v \sqrt{v+\beta}}{(u-v)(u-2-v)}+\frac{u \sqrt{u+\beta}-(v-2) \sqrt{v-2+\beta}}{(u-v)(u-v+2)} ;
\end{aligned}
$$




$$
\begin{aligned}
& X_{(2)}=-\frac{u v \sqrt{u-2+\beta}-u v \sqrt{v+\beta}}{(u-v)(u-2-v)}+\frac{u v \sqrt{u+\beta}-u v \sqrt{v-2+\beta}}{(u-v)(u-v+2)}, \\
& Y_{(2)}=\frac{v \sqrt{u-2+\beta}-v \sqrt{v+\beta}}{(u-v)(u-2-v)}-\frac{u \sqrt{u+\beta}-u \sqrt{v-2+\beta}}{(u-v)(u-v+2)} .
\end{aligned}
$$

and the functions $X_{(3)}, Y_{(3)}, X_{(4)}, Y_{(4)}$ are expressed in a similar way. Since $b_{n}=a_{n}^{*} a^{2}+2 a_{n}$, the operator $z_{n}=X_{N} a_{n}+Y_{N} b_{n}$ can be rewritten in the form (3.15) with

$$
\tilde{X}_{N}=X_{(1)}+X_{(2)}+2\left(Y_{(1)}+Y_{(2)}\right), \quad \tilde{Y}_{N}=Y_{(1)}+Y_{(2)}
$$

Then, Eqs. (A.13)-(A.14) yield

$$
\begin{aligned}
& \tilde{X}_{N}=\frac{(u-2) \sqrt{u-2+\beta}-(v-2) \sqrt{v-2+\beta}}{u-v}, \\
& \tilde{Y}_{N}=-\frac{\sqrt{u-2+\beta}-\sqrt{v-2+\beta}}{u-v} .
\end{aligned}
$$

and passing back from $(u, v)$ to $(H, J)$, we arrive at (3.16).

The commutator $\left[z_{m}, z_{n}\right]$ is obtained by anti-symmetrization of

$$
z_{m} z_{n}=X_{N} a_{m} a_{n} \tilde{X}_{N}+X_{N} a_{m} a_{n}^{*} a^{2} \tilde{Y}_{N}+Y_{N} a^{2} a_{m}^{*} a_{n} \tilde{X}_{N}+Y_{N} a^{2} a_{m}^{*} a_{n}^{*} a^{2} \tilde{Y}_{N}
$$

where the representations (3.7) and (3.15) are used for $z_{m}$ and $z_{n}$ respectively. Taking into account that the operator $J_{m n}=i\left(a_{n}^{*} a_{m}-a_{m}^{*} a_{n}\right)$ commutes with scalar operators and that $X_{N}\left(H, J^{2}\right) a^{2}=a^{2} X_{N}\left(H-2, J^{2}\right)$, the commutator $\left[z_{m}, z_{n}\right]$ reduces to (3.20).

To represent the commutator $\left[z_{m}^{*}, z_{n}\right]$ in the form (3.21) we also use the exchange relations of $a_{n}^{*}$ and $b_{n}^{*}$ with scalar operators

$$
\begin{aligned}
& f(u, v) a_{n}^{*}=a_{n}^{*} \frac{u f(u+2, v)-v f(u, v+2)}{u-v}-b_{n}^{*} \frac{f(u+2, v)-f(u, v+2)}{u-v}, \\
& f(u, v) b_{n}^{*}=a_{n}^{*} \frac{u v f(u+2, v)-u v f(u, v+2)}{u-v}-b_{n}^{*} \frac{v f(u+2, v)-u f(u, v+2)}{u-v},
\end{aligned}
$$

which can be derived similarly to (A.10). Writing the terms $z_{m}^{*} z_{n}$ and $z_{n} z_{m}^{*}$ as

$$
\begin{aligned}
& z_{m}^{*} z_{n}=\left(a_{m}^{*} X_{N}+b_{m}^{*} Y_{N}\right)\left(a_{n}\left(\tilde{X}_{N}-2 \tilde{Y}_{N}\right)+b_{n} \tilde{Y}_{N}\right), \quad \text { and } \\
& z_{n} z_{m}^{*}=\left(a_{n} \tilde{X}_{N}+a_{n}^{*} a^{2} \tilde{Y}_{N}\right)\left(a_{m}^{*} X_{N}+b_{m}^{*} Y_{N}\right)
\end{aligned}
$$

respectively, and applying (A.10), (A.19) and then (3.1), we obtain the commutator $\left[z_{m}^{*}, z_{n}\right]$ in the form (3.21). 


\section{References}

[1] G. Jorjadze, L. O'Raifeartaigh, I. Tsutsui, Phys.Lett. B 336 (1994) 388, hep-th/9407059

G. Jorjadze, W. Piechocki, Phys. Lett. B 448 (1999) 203, hep-th/9812199.

Phys. Lett. B 461 (1999) 183, gr-qc/9811094.

[2] T. Fulop, J.Math.Phys. 38 (1997) 61, hep-th/9602040

J.Math.Phys. 37 (1996) 1617, hep-th/9502145.

[3] J. Lucietti, JHEP 0305 (2003) 017, hep-th/0303228.

[4] H. Dorn, G. Jorjadze, Fortsch. Phys. 53 (2005) 486, hep-th/0502081.

[5] O. Aharony, S.S. Gubser, J. M. Maldacena, H. Ooguri, Y. Oz, Phys.Rept. 323 (2000) 183, hep-th/9905111.

[6] E. D'Hoker, D.Z. Freedman, "Supersymmetric Gauge Theories and the AdS/CFT Correspondence", hep-th/0201253.

[7] H. Dorn, M. Salizzoni and C. Sieg, JHEP 0502 (2005) 047, hep-th/0307229

[8] D. Berenstein, J. M. Maldacena and H. Nastase, JHEP 0204 (2002) 013 arXiv:hep-th/0202021.

[9] H. Dorn and G. Jorjadze, "Massless scalar particle on AdS spacetime: Hamiltonian reduction and quantization," arXiv:hep-th/0508072.

[10] H.A. Kastrup, Fortsch. Phys. 51 (2003) 975, quant-ph/0307069.

[11] S. Minwalla, Adv. Theor. Math. Phys. 2 (1998) 781, hep-th/9712074.

[12] T. Holstein and H. Primakoff. Phys. Rev. 58 (1940) 1098.

[13] M. Gunaydin and C. Saclioglu, Commun.Math.Phys. 87 (1982) 159.

[14] C. Fronsdal, Phys. Rev. D 10 (1974) 589.

M. Flato and C. Fronsdal, Lett. Math. Phys. 2 (1978) 421. 